\title{
Change in body surface temperature as an ancillary measurement to motor evoked potentials
}

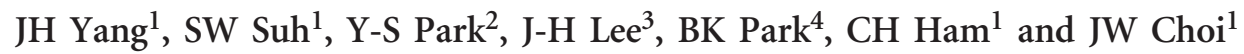

Study design: Experimental study.

Objectives: To study the role of surface temperature as an adjunct to motor evoked potentials (MEPs) in rabbit spinal cord injury (SCl) model.

Setting: Department of Orthopedics, Korea University Guro Hospital, Seoul, Korea.

Methods: Rabbits $(n=18)$ were divided into Complete $(n=9)$ and Incomplete $(n=9) \mathrm{SCl}$ groups. Complete SCl was defined as being non-responsive to a wake-up test with loss of MEPs after transection of spinal cord. Incomplete SCl was defined as being responsive to a wake-up test with significant attenuation $(\geqslant 80 \%)$ of MEPs after impaction on spinal cord. Surface temperature of upper and lower extremities, core temperature and MEPs signals were checked before, during and after $\mathrm{SCl}$ for $20 \mathrm{~min}$. A wake-up test was conducted and spinal cord was histologicaly evaluated.

Results: Experimental conditions between the two groups were statistically similar ( $P>0.005$ for all values). After $\mathrm{SCl}$, upper extremity temperatures did not change in either group $(P>0.005)$; however, the surface temperature of the lower extremities in the Complete SCl Group elevated to $1.7 \pm 0.5^{\circ} \mathrm{C}$ in comparison to $0.5 \pm 0.1{ }^{\circ} \mathrm{C}$ in the Incomplete $\mathrm{SCl}$ Group $(P<0.001)$. The scores of wake-up test in the Incomplete SCI Group were significantly different from that of the Complete $\mathrm{SCl}$ Group $(P<0.001)$, while white and gray matter damage was variable on histology.

Conclusions: Monitoring of changes of body surface temperature of the lower extremities can be potentially used to identify the completeness of $\mathrm{SCl}$ in a rabbit model.

Spinal Cord (2015) 53, 827-834; doi:10.1038/sc.2015.90; published online 30 June 2015

\section{INTRODUCTION}

The recent advances in intraoperative neurophysiological monitoring have come as a boon to spine surgeons, by adding strength to our ability to deal with complex spine pathologies such as deformities and tumors. ${ }^{1}$ Motor evoked potential (MEP) has been widely used as an intraoperative neurophysiologic monitoring system. It measures the real-time electrical activity of the spinal cord during spine surgeries, in which spinal cord injury (SCI) may occur. Despite their popularity, its utility is limited by several factors such as the likelihood of false-negative and false-positive results, age of the patient, type of diseases and surgical procedures, electronic equipment and type of anesthesia. ${ }^{2-4}$ Moreover, MEP signals have been known to disappear even with trivial trauma that is insufficient to cause clinically significant SCI. Also, MEP monitoring cannot predict the depth of SCI (complete or incomplete), based on the amount of decrement in amplitude. These shortfalls of MEP monitoring can make spine surgeons not to assess the status of the spinal cord, which is crucial for the intraoperative decisions for surgical treatment.

To overcome the shortfalls of MEP monitoring, wake-up test still continue to serve as useful adjunct. ${ }^{1}$ However, the wake-up test significantly distresses the patient both physically and psychologically.
In view of anesthetists, it is technically difficult to perform. Therefore, it cannot be performed as frequently as the surgeon wishes to. Such disadvantages render it clinically unfavorable and support the use of MEP. Meanwhile, the authors have observed intraoperative surface temperature changes in the lower limb, in response to the SCI, during spinal deformity corrections and tumor resections.

Therefore, in this study, body surface temperature was hypothesized as a modality that indicates the completeness of SCI, since it is known to change in response to SCI as a result of autonomic dysfunction. With this in the background, the role of monitoring temperature changes of the lower extremity was evaluated, as an ancillary tool to MEP for assessing the extent of SCI during spine surgeries.

\section{MATERIALS AND METHODS}

This study was performed under the approval of Institutional Animal Care and Use Committee (KU12129). Thirty-five 3-month-old male New Zealand white breed rabbits weighing $\sim 3 \mathrm{~kg}$ were used, due to their proper size, and as they are easy to handle, and house bred. Furthermore, it is a well-established model that is well recognized by the scientific and regulatory communities. Three-month-old male rabbits are considered equivalent to a 20 -year-old human. Considering the susceptibility and the physiologic influence during

${ }^{1}$ Department of Orthopedics, Scoliosis Research Institute, Korea University Medical College, Guro Hospital, Seoul, Korea; ${ }^{2}$ Department of Orthopedics, Hanyang University, Guri Hospital, Guri, Korea; ${ }^{3}$ Department of Orthopedics, Kyung-hee University Medical Center, Seoul, Korea and ${ }^{4}$ Department of Physical Medicine and Rehabilitation, Korea University Medical College, Ansan Hospital, Ansan-si, Korea

Correspondence: Professor SW Suh, Department of Orthopedics, Scoliosis Research Institute, Korea University Medical College, Guro Hospital, \#80, Guro-dong, Guro-Gu, Seoul 152-703, Korea.

E-mail: spine@korea.ac.kr

Received 4 August 2014; revised 19 February 2015; accepted 5 March 2015; published online 30 June 2015 
changing rabbit warren, a 10-day period of adjustment was provided prior to the start of the experiment.

Rabbits were divided into a Complete SCI group (14 cases) and an Incomplete SCI Group (21 cases). The Complete SCI Group was defined as following: complete loss of MEP signal, no spontaneous response on wake-up test and no response to Von Frey monofilament test (Stoelting, Wood Dale, IL, USA) under $180 \mathrm{~g}$ of pressure on arousal. ${ }^{5,6}$ The Incomplete SCI Group was defined as following: attenuation of MEPs signal amplitude by $>80 \%$ compared with baseline potential, spontaneous response of lower extremities on wake-up test and response under Von Frey monofilament test at $180 \mathrm{~g}$ of pressure. ${ }^{5,6}$ Considering the shortcoming of a single monitoring with muscular MEP, such as instability and high sensitivity of baseline amplitude, the amplitude of MEP in subjects was measured at 3-min intervals. ${ }^{7}$ If there were any recovery of MEP amplitude, the subject was excluded from the Incomplete group, as well as from this study. For the assessment of the wake-up test, anesthesia was discontinued until the return of free movements of the head and stable vital signs. Subsequently, threatening hand movements were exposed in front of the subject's eyes three times at 3-min intervals. If the subject failed to show any movement in the hind foot, it was recorded as positive. For the Von Frey monofilament test, $180 \mathrm{~g}$ Von Frey monofilament was applied five times perpendicularly to the underside of the paw of each animal for 2-3 s. Response to weight more than three out of five times was recorded as positive. ${ }^{8}$

\section{Anesthesia and MEP application}

The experiment was conducted on each rabbit on a prone position. A vascular catheter was placed in the left ear vein followed by an induction and maintenance (12-20 $\mathrm{mg} \mathrm{kg}^{-1} \mathrm{~h}^{-1}$ ) using intravenous anesthetics (Propofol,
Astra-Zeneca, Luton, UK). Oxygen saturation, pulse rate, arterial blood pressure (artery of the contralateral ear), $\mathrm{CO} 2$ partial pressure and core temperature were measured using a $5 / \mathrm{S}$ Avance anesthesia device (DatexOhmeda, Madison, WI, USA) (Figure 1). The core temperature was measured at rectum $30-\mathrm{cm}$ deep into the anus using a flexible thermometer (GeneralPurpose Temperature Probe; DeRoyal Industries Inc, Powell, TN, USA). Following the anesthesia, MEP monitoring equipment (Nims Spine System, Medtronic Sofamor Danek, Memphis, TN, USA) providing a constant current stimulus that is set to $250 \mu \mathrm{s}$ of pulse width, $4 \mathrm{~ms}$ of inter-stimulus interval and $5-\mathrm{Hz}$ repetition rate was installed. ${ }^{7}$ Stimulators of MEPs were placed on the rabbit's head (forehead and mandible) and needles as input devices were inserted at $>3-\mathrm{cm}$ apart on the Rectus femoris muscles (Figure 2). ${ }^{9}$ A biphasic square pulse wave was used as the input signal. Stimulus was started at $20 \mathrm{~mA}$ and was gradually increased to $60 \mathrm{~mA}$ by increments of $10 \mathrm{~mA}$. The upper limit was set at $60 \mathrm{~mA}$ to avoid possible side effects. ${ }^{10}$ When proper signals were obtained, it was measured three times at 3-min intervals and the mean value measured was recorded as baseline potential. ${ }^{9}$

\section{Measurement of body surface temperature}

Considering the susceptibility of body surface temperature to laboratory temperature and humidity, they were maintained at $24^{\circ} \mathrm{C}$ and $40 \%$, respectively, with a single separated air conditioning device (with $2{ }^{\circ} \mathrm{C}$ and $10 \%$ error margin). Sensors for the body surface temperature were inserted subdermally. Body surface temperature was measured behind the calves and anterior elbows for the lower and the upper extremities, respectively.

Temperature sensors (SENTECH, Seoul, Korea), with measurement range of -200 to $500{ }^{\circ} \mathrm{C}$, that can detect a change of temperature as little as one decimal
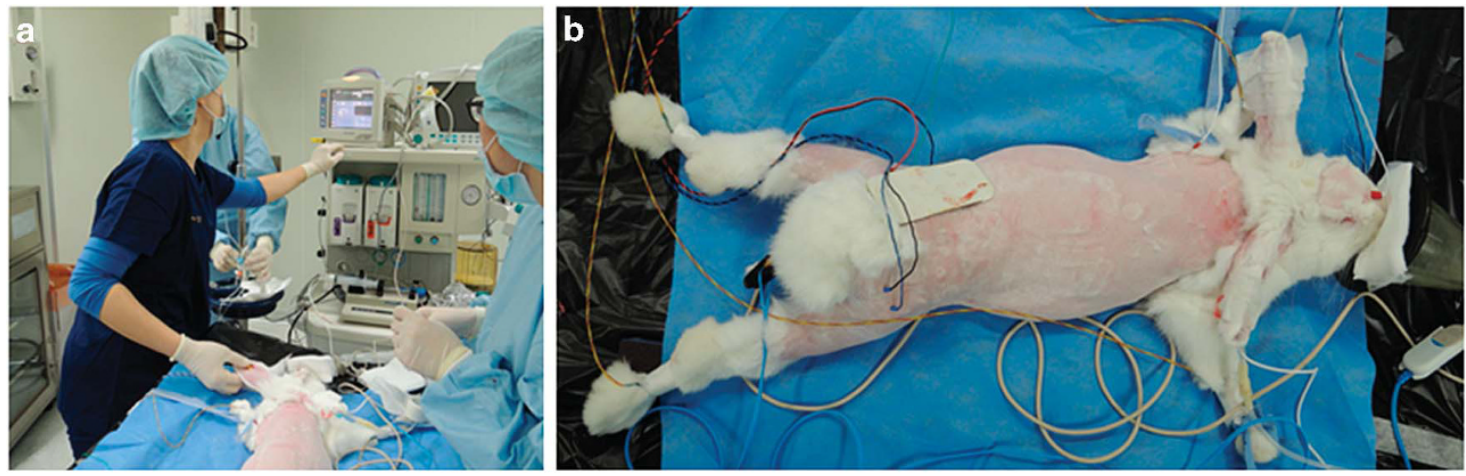

Figure 1 (a) Oxygen saturation, pulse rate, arterial blood pressure (artery of the contralateral ear) and carbon dioxide partial pressure were measured while maintaining anesthesia by injecting Propofol. (b) Motor evoked potential signals were identified in bilateral quadriceps femoris muscles with the assistance of veterinarians, and the body surface temperatures were measured in both lower and upper extremities.
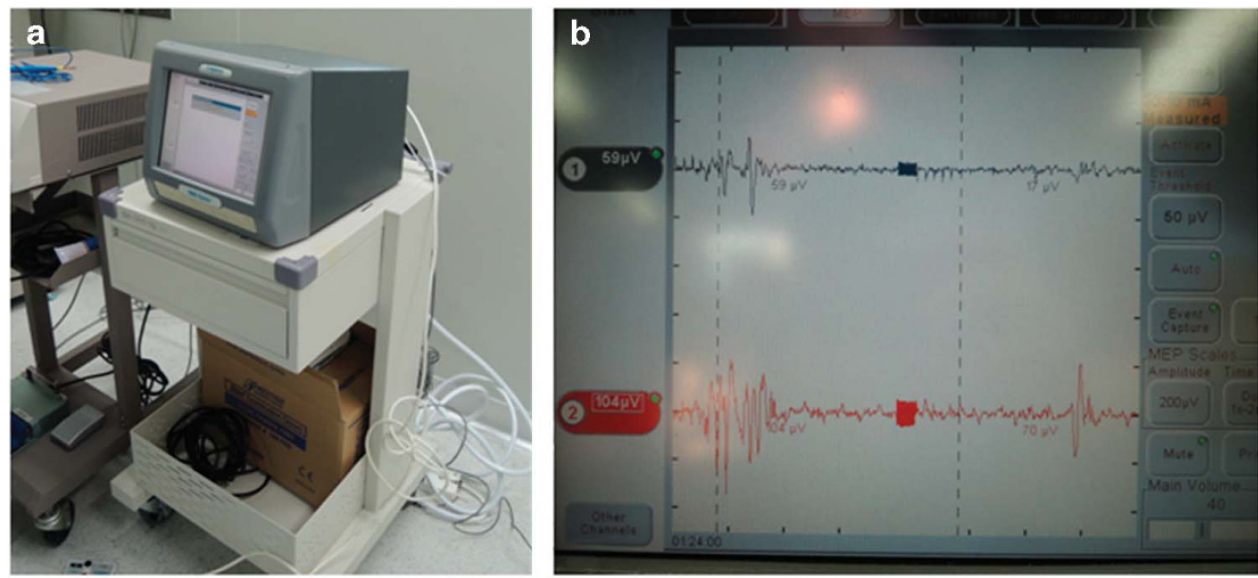

Figure 2 (a) TES-MEP equipment (Nims Spine System) was used in the experiment. (b) MEP was measured consistently in the quadriceps muscle, considering the positions of the rabbit's lower extremity muscles, and two hypodermic needles were inserted 3-cm apart. 


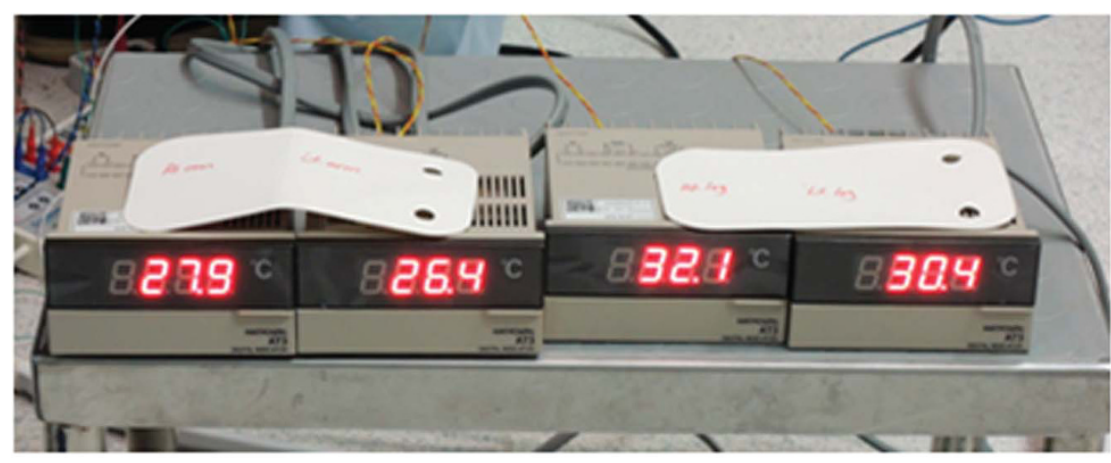

Figure 3 The body surface temperature measuring devices (SENTECH) were used in the experiment. The two devices on the left indicate the body surface temperature of the upper extremities, and the other two on the right indicate the body surface temperature of the lower extremities.
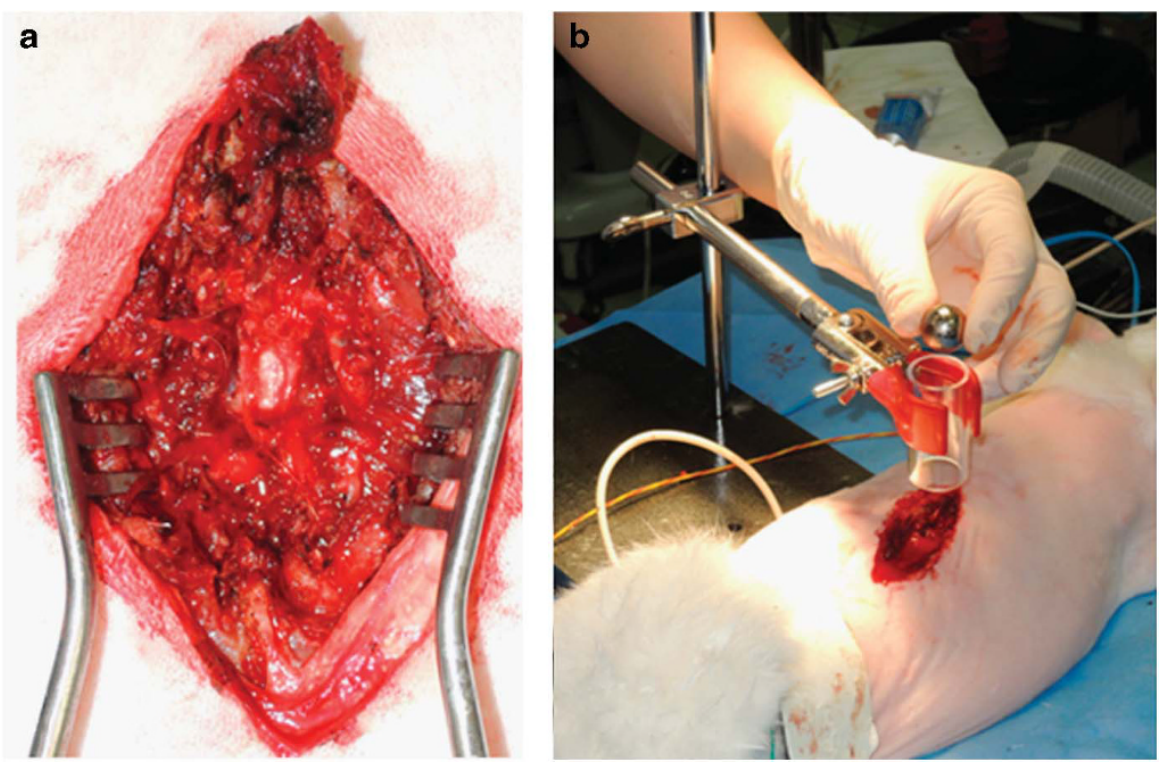

Figure 4 The incomplete spinal cord injury model. (a) Posterior arch of the vertebra was completely removed and the spinal cord was exposed. (b) After exposing the spinal cord, direct impact was applied on the spinal cord by using impact tool (Shinwoo company) from a height of $3 \mathrm{~cm}$.

place with accuracy of $\pm 0.15^{\circ} \mathrm{C}$ (Figure 3) were used. Body surface temperature was measured 10 times at an interval of $30 \mathrm{~s}$ for $5 \mathrm{~min}$ prior to the experiment. The average temperature was defined as the baseline temperature before the experiment.

\section{SCI model preparation}

The T7-8 vertebral junction was selected as the site of injury, as SCI in this region can lead to autonomic dysfunction without affecting the cardio-vascular function, which may influence signals of transcranial electrical stimulationmotor evoked potential (TES-MEP) by altering the blood pressure. ${ }^{11}$ The location of the T7-8 vertebral junction was confirmed using mobile radiography equipment (C-arm) or general radiography equipment (Figure 4). Different methods of spinal cord damage were used for each SCI groups, to clearly categorize the SCI groups and to avoid errors that may arise from the spinal shock. For the Incomplete SCI Group, weight drop method was used to create injury, as it exhibits similar mechanism to the injury seen in clinical situations. ${ }^{12}$ Conversely, since SCI created by weight drop method preserved conductive function, ${ }^{13}$ for the complete SCI model the spinal cord was completely transected to eliminate voluntary movements. For the incomplete injury created by impaction weight drop, the outer cortex of the lamina of T7-8 was ground using a motorized drill leaving a paper-thin lamina. Subsequently, a 9.5-g-weighted iron pendulum, $12 \mathrm{~mm}$ in diameter (Shinwoo Company, Inchun, Korea) was impacted. The magnitude of impact was adjusted by changing the height of the pendulum. Originally, the amount of impact was determined based on the previous study by Reece TB et al., ${ }^{14}$ who developed a $10 \mathrm{~g}$ weight drop from a height of $60 \mathrm{~mm}$ (Figure 4). However, in our experiment it was difficult to reproduce consistent incomplete SCI, due to variable impact or individual differences on resistance of the cord to the impact. Therefore, impact force was reduced by half by dropping the pendulum from a 30-mm height after completely exposing the spinal cord without lamina. After the impaction on the cord, MEP and wake-up tests were subsequently done. Thereafter, to rule out spinal shock in Incomplete SCI Group, the animal that did not present any decrease in MEP signal by $>80 \%$ or not showing any response to the wake-up test was excluded from the experiment.

The procedure for complete SCI was as follows: laminae of T7-8 were completely removed using a motorized drill and a 1-mm Kerrison punch (Aesculap, Inc., Center Valley, PA, USA) under MEP neuromonitoring throughout the entire process. After the exposure of the spinal cord, the body surface temperature was measured. If the temperature difference was higher than $2{ }^{\circ} \mathrm{C}$ in comparison to the baseline, temperature was measured an additional four times at 5-min intervals. If the difference of temperature in additional measurements continued to be $>2{ }^{\circ} \mathrm{C}$, the subject animal was excluded from the experiment, as the previous study suggested that the temperature difference of $2{ }^{\circ} \mathrm{C}$ was a sign of homeostasis breakdown. ${ }^{15} \mathrm{~A}$ complete transection of the spinal cord was made using a number 15 scalpel. The MEP was measured immediately after the transection of the spinal cord and was measured five times in 3-min intervals. 

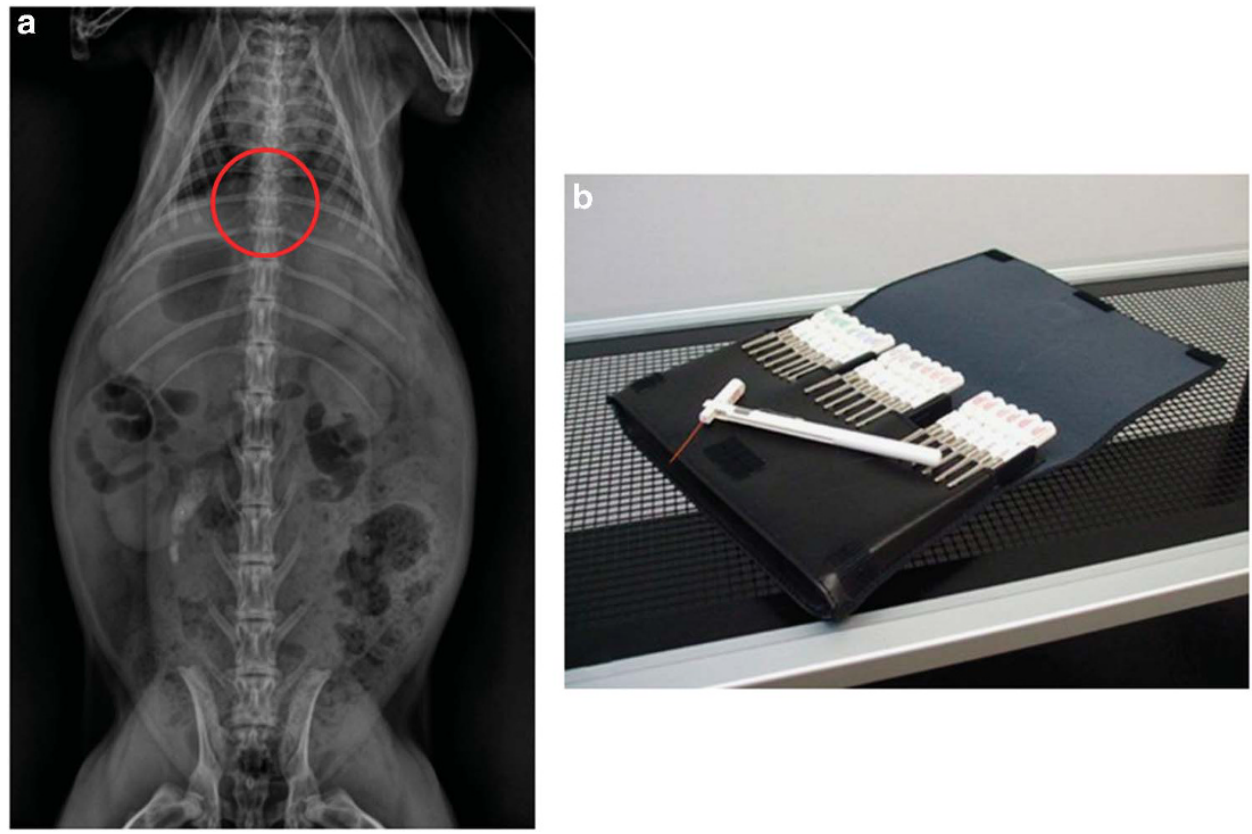

Figure 5 (a) The position of T7-8 was confirmed on radiologic scanning before the experiment. (b) Response test was implemented using Von Frey monofilament as the judgment standard of complete and incomplete injury after the experiment.

Table 1 Demographic information of the complete injury group

\begin{tabular}{|c|c|c|c|c|c|c|c|c|c|c|c|c|c|c|}
\hline \multirow[t]{2}{*}{ No } & \multirow[t]{2}{*}{ Sex } & \multirow[t]{2}{*}{ Age (month) } & \multirow{2}{*}{ Weight (kg) } & \multirow{2}{*}{ Room temp $\left({ }^{\circ} \mathrm{C}\right)$} & \multirow{2}{*}{ Humidity (\%) } & \multicolumn{3}{|c|}{ Core body temperature } & \multicolumn{2}{|c|}{ Wake-up test } & \multicolumn{2}{|l|}{$\mathrm{SpO}_{2}$} & \multicolumn{2}{|l|}{$\mathrm{EtCO}_{2}$} \\
\hline & & & & & & Base & Impact & Final & Rt. & $L t$. & Pre & Post & Pre & Post \\
\hline 1 & $\mathrm{M}$ & 4 & 3 & 23.3 & 37 & 38.1 & 37.9 & 38.3 & 0 & 0 & 99 & 98 & 36 & 36 \\
\hline 2 & $M$ & 4 & 3 & 26.1 & 39 & 38.9 & 38.5 & 39.0 & 0 & 0 & 98 & 97 & 40 & 34 \\
\hline 3 & $M$ & 4 & 3 & 22.8 & 45 & 37.5 & 37.8 & 37.9 & 0 & 0 & 100 & 98 & 35 & 39 \\
\hline 4 & $M$ & 4 & 3 & 21.8 & 45 & 38.0 & 37.9 & 38.1 & 0 & 0 & 100 & 99 & 36 & 35 \\
\hline 5 & $M$ & 4 & 3 & 23.9 & 45 & 39.2 & 39.0 & 39.1 & 0 & 0 & 99 & 97 & 37 & 36 \\
\hline 6 & M & 4 & 3 & 22.3 & 45 & 36.9 & 37.2 & 37.3 & 0 & 0 & 100 & 99 & 34 & 36 \\
\hline 7 & $M$ & 4 & 3 & 21.7 & 46 & 37.9 & 38.0 & 38.1 & 0 & 0 & 99 & 99 & 37 & 39 \\
\hline 8 & $M$ & 4 & 3 & 21.7 & 46 & 39.4 & 39.1 & 39.5 & 0 & 0 & 98 & 100 & 39 & 40 \\
\hline 9 & $M$ & 4 & 3 & 22.7 & 46 & 38.5 & 38.8 & 38.9 & 0 & 0 & 99 & 100 & 39 & 34 \\
\hline Mean & & 4.0 & 3.0 & 22.9 & 43.8 & 38.3 & 38.2 & 38.3 & 0.0 & 0.0 & 99.1 & 98.6 & 37.0 & 36.6 \\
\hline s.d. & & 0.0 & 0.0 & 1.4 & 3.3 & 0.8 & 0.6 & 0.7 & 0.0 & 0.0 & 0.8 & 1.1 & 2.0 & 2.2 \\
\hline Statistical analysis & & & & & & & $P=0.549^{a}$ & & $P=1.00^{b}$ & & $P=0.248^{\mathrm{b}}$ & & $P=0.888^{\mathrm{b}}$ & \\
\hline
\end{tabular}

Abbreviations: Lt., left; M, male; Rt. right.

${ }^{\text {a}}$ For statistical analysis, Friedman test was used. $P$-values $<0.05$ were considered significant.

${ }^{b}$ For statistical analysis, Wilcoxon signed-rank test was used. $P$-values $<0.05$ were considered significant.

After a complete or an incomplete SCI, body surface temperature was measured 60 times in 20 min with 20-s intervals. Motor function of the lower extremities was assessed with the wake-up test. After the experiment, to uniformly and easily assess the post injury motor function in all experimental cases, a modified Tarlov and BBB scale (Basso, Beattie and Bresnahan Locomotor Rating Scale) was applied. ${ }^{16,17}$ A score of 2 points was given in case of spontaneous movement; 1 point was given if movement was observed during the Von Frey filament test; and 0 points were given if no movement was observed (Figure 5). Due to ethical problems according to regulation guidelines of the IACUC, biological weakness of rabbit after the experiment and limitation of experiment budget, clinical observation was done only on acute state after wakeup. After the completion of the experiment, the spinal cord was extracted $1 \mathrm{~cm}$ above and below the impacted area, followed by the fixation of the specimen with formalin solution. Histological evaluation was only done in the Incomplete SCI Group. By contrast, it was not done in the Complete SCI
Group as the spinal cord was definitely transected in the latter group. The harvested spinal cord was dyed with Luxor fast blue and hematoxylin and eosin (both purchased from EMD Chemical Ltd, Philadelphia, PA, USA). Histological analysis was carried out with an axial slice along the damaged spinal cord portion and the extent of injury was observed.

\section{Statistical analysis}

A Mann-Whitney $U$-test, a Wilcoxon signed-rank test and a Friedman test were used for non-parametric comparative analysis of average values, as described in Tables 1-3. For the statistical analysis, SPSS software (version 18.0, IBM, Armonk, NY, USA) was used and $P$-values $<0.05$ were considered statistically significant. Owing to the small sample size, statistical values were re-verified using the G-power 3.1 program. ${ }^{18}$ If the power of a statistical value was $>0.8$, it was considered highly significant. The statistical results were described with $P$-values (power of statistical results). 
Table 2 Demographic information of the Incomplete injury group

\begin{tabular}{|c|c|c|c|c|c|c|c|c|c|c|c|c|c|c|}
\hline \multirow[t]{2}{*}{ No } & \multirow[t]{2}{*}{ Sex } & \multirow[t]{2}{*}{ Age (month) } & \multirow[t]{2}{*}{ Weight (kg) } & \multirow[t]{2}{*}{ Room temp $\left({ }^{\circ} \mathrm{C}\right)$} & \multirow[t]{2}{*}{ Humidity (\%) } & \multicolumn{3}{|c|}{ Core body temperature } & \multicolumn{2}{|c|}{ Wake-up test } & \multicolumn{2}{|c|}{$\mathrm{SpO}_{2}$} & \multicolumn{2}{|c|}{$\mathrm{EtCO}_{2}$} \\
\hline & & & & & & Base & Impact & Final & Rt. & $L t$. & Pre & Post & Pre & Post \\
\hline 2 & M & 4 & 3 & 22.0 & 45 & 37.9 & 37.8 & 38.1 & 1 & 1 & 100 & 98 & 37 & 36 \\
\hline 3 & M & 4 & 3 & 23.7 & 45 & 39.7 & 39.5 & 39.7 & 2 & 1 & 98 & 99 & 39 & 39 \\
\hline 4 & M & 4 & 3 & 23.0 & 45 & 37.9 & 37.9 & 38.0 & 1 & 1 & 100 & 99 & 35 & 40 \\
\hline 7 & M & 4 & 3 & 22.2 & 47 & 39.3 & 39.5 & 39.1 & 1 & 1 & 99 & 97 & 40 & 36 \\
\hline 8 & M & 4 & 3 & 22.2 & 47 & 37.9 & 37.9 & 38.1 & 1 & 0 & 98 & 100 & 36 & 40 \\
\hline 9 & M & 4 & 3 & 23.3 & 47 & 37.8 & 37.9 & 38.0 & 2 & 1 & 99 & 98 & 36 & 37 \\
\hline Mean & & 4.0 & 3.0 & 22.9 & 45.8 & 38.4 & 38.3 & 38.3 & 1.4 & 1.1 & 99.1 & 98.9 & 36.8 & 37.2 \\
\hline s.d. & & 0.0 & 0.0 & 0.7 & 1.4 & 0.8 & 0.8 & 0.7 & 0.5 & 0.6 & 0.8 & 1.1 & 1.7 & 2.4 \\
\hline
\end{tabular}

Abbreviations: Lt., left; M, male; Rt. right.

${ }^{a}$ For statistical analysis, Friedman test was used. $P$-values $<0.05$ were considered significant.

bFor statistical analysis, Wilcoxon signed-rank test was used. $P$-values $<0.05$ were considered significant.

\section{a}

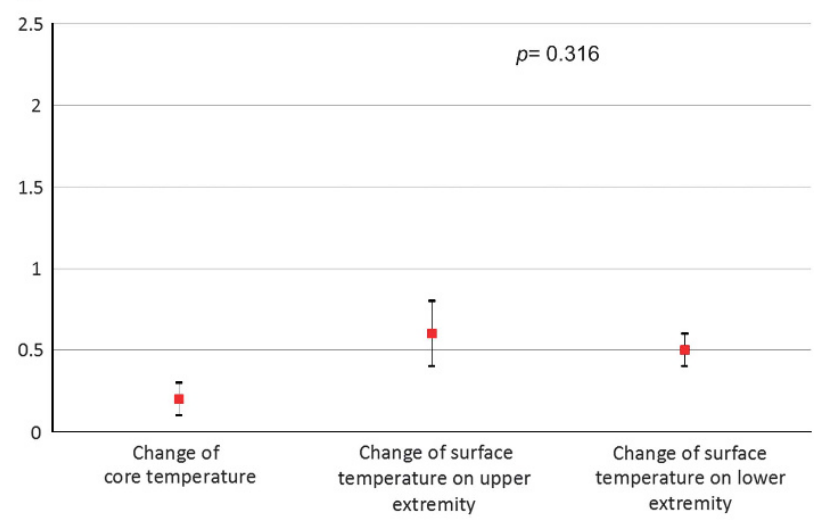

b

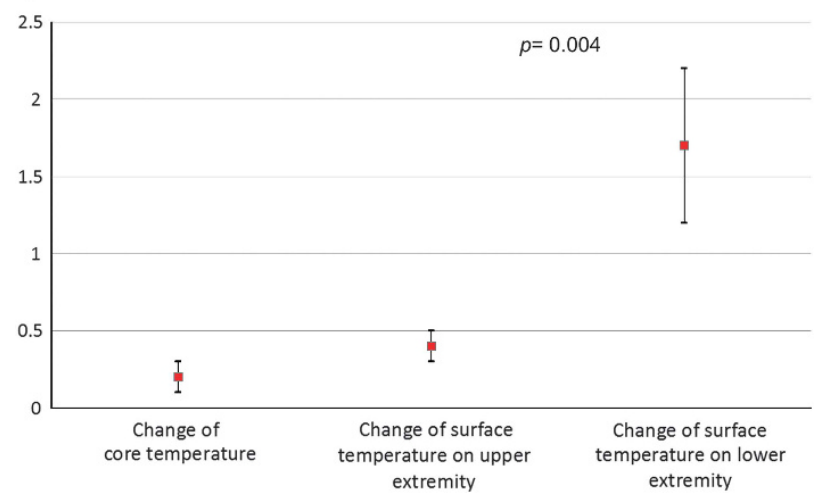

Figure 6 (a) Changes of body surface temperature in the upper and lower extremities vs elapsed time in the Incomplete SCI Group after the injury. In the lower extremity, increased body surface temperature compared with that of the upper extremity is noticeable. (b) Changes of body surface temperature in the upper and lower extremities vs elapsed time in the Complete $\mathrm{SCl}$ Group after the injury. It is noticeable that the body surface temperature change in the lower extremity is subtle, and this is the same modality as that of the body surface temperature change in the upper extremity.
Table 3 Comparison between Complete SCI Group and Incomplete $\mathrm{SCl}$ Group according to evaluated factors

\begin{tabular}{lccc}
\hline Factors & $\begin{array}{c}\text { Complete } \\
\text { injured group }\end{array}$ & $\begin{array}{c}\text { Incomplete } \\
\text { injured group }\end{array}$ & P value \\
\hline Age (month) & $4.0 \pm 0.0$ & $4.0 \pm 0.0$ & 1.00 \\
Weight (kg) & $3.0 \pm 0.0$ & $3.0 \pm 0.0$ & 1.00 \\
Room temperature $\left({ }^{\circ} \mathrm{C}\right)$ & $22.9 \pm 1.4$ & $22.9 \pm 0.7$ & 0.685 \\
Room humidity $(\%)$ & $43.8 \pm 3.3$ & $45.8 \pm 1.4$ & 0.118 \\
Core body temperature $\left({ }^{\circ} \mathrm{C}\right)$ & $38.3 \pm 0.8$ & $38.4 \pm 0.8$ & 0.929 \\
Change in surface temperature & $0.4 \pm 0.1$ & $0.6 \pm 0.2$ & 0.094 \\
$\left({ }^{\circ} \mathrm{C}\right.$, upper extremity) & $1.7 \pm 0.5$ & $0.5 \pm 0.1$ & $<0.001(0.99)$ \\
Change in surface temperature & & & \\
$\left({ }^{\circ} \mathrm{C}\right.$, lower extremity) & $0.0 \pm 0.0$ & $2.6 \pm 0.9$ & $<0.001$ \\
Wake-up test & & & \\
\hline
\end{tabular}

Abbreviation: $\mathrm{SCl}$, spinal cord injury.

aFor statistical analysis, Mann-Whitney $U$-test was used. $P$-values $<0.05$ were considered aFor statistical
significant.

\section{RESULTS}

Seventeen subjects were excluded from the experiment due to the accidental SCI during the surgical procedure, dissipation of evoked potential, or errors in measuring position or the death of the animal. The Complete and the Incomplete SCI Groups consisted of nine cases each. Laboratory temperatures were $22.9 \pm 1.1^{\circ} \mathrm{C}$; and the humidity was $44.8 \pm 2.7 \%$; oxygen saturation values were $99.1 \pm 0.8 \%$ and $98.7 \pm 1.1 \%$ before and after the experiment, respectively. Systolic and diastolic arterial blood pressure before the experiment were $85.1 \pm 2.3 \mathrm{~mm} \mathrm{Hg}$ and $60.8 \pm 7.6 \mathrm{~mm} \mathrm{Hg}$, respectively, and after the experiment $84.7 \pm 1.5 \mathrm{mmHg}$ and $60.8 \pm 3.4 \mathrm{~mm} \mathrm{Hg}$, respectively. Carbon dioxide concentration was measured as $36.9 \pm 1.8$ before and $36.9 \pm 2$ after the experiment. The core temperature was measured as $38.3 \pm 0.8^{\circ} \mathrm{C}$ before the experiment, $38.3 \pm 0.7^{\circ} \mathrm{C}$ after the impact and $38.3 \pm 0.7^{\circ} \mathrm{C}$ after the experiment. Tables 1 and 2 depict the experimental data of each animal. 

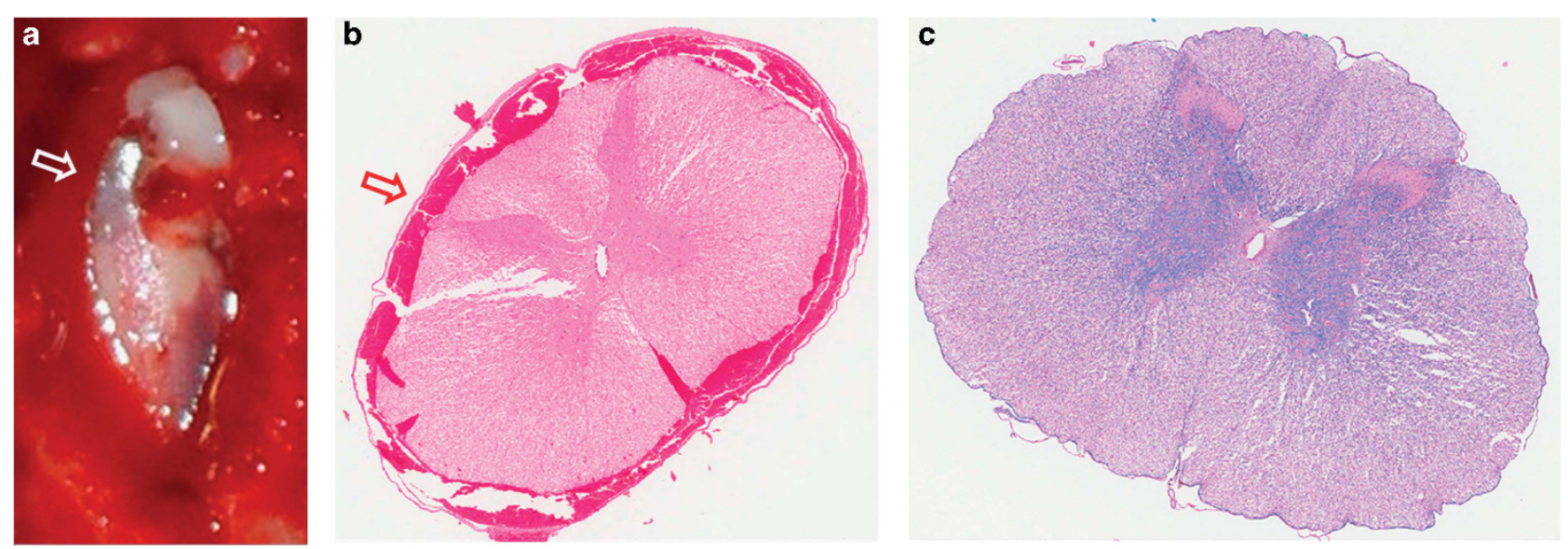

Figure 7 (a) The subdural hematoma observed in the Incomplete SCl cases under direct vision (white arrow) in a complete spinal cord injury case. (b) Microscopic subdural hematoma can be seen, but neural cell breakage was not observed in the anterior horn of gray matter, in which the autonomic nervous system is located, in contrast with breakage in neural connective tissue around the lateral funiculus. Other inflammatory cells were not found, due to induced euthanasia and formalin fixing done before secondary inflammation reaction could occur. (c) In another section without hemorrhage, neural cell breakage was not observed in the anterior horn of gray matter on both sides; however, breakage in neural connective tissue around the lateral funiculus in white matter, in which the cortico-spinal tract was located, was observed.

\section{Change in body surface temperature associated with SCI}

All cases of the Complete SCI Group demonstrated an increase in surface body temperature after the transection of the spinal cord, with mean magnitude change of $1.7 \pm 0.5^{\circ} \mathrm{C}$ in the lower extremities $\left(1.7 \pm 0.4^{\circ} \mathrm{C}\right.$ on the right and $1.7 \pm 0.7^{\circ} \mathrm{C}$ on the left side) and $0.4 \pm 0.1{ }^{\circ} \mathrm{C}$ in upper extremities $\left(0.4 \pm 0.2^{\circ} \mathrm{C}\right.$ on the right upper and $0.4 \pm 0.1^{\circ} \mathrm{C}$ on the left upper extremity). The mean change in core temperature was $0.2 \pm 0.1^{\circ} \mathrm{C}$. Statistically, the magnitude of temperature change in the lower extremities was significantly different from the temperature changes of the upper extremities and core temperature $(P=0.004$ (1.00) and $P=0.002$ (1.00), respectively) (Figure 6a).

By contrast, the Incomplete SCI Group did not show any increase in temperature of the lower extremities in any cases. After the impact, the mean magnitude of temperature change in the lower extremities was $0.5 \pm 0.1{ }^{\circ} \mathrm{C}$ (right side $0.5 \pm 0.2^{\circ} \mathrm{C}$ and left side $0.6 \pm 0.2^{\circ} \mathrm{C}$ ) and $0.6 \pm 0.2^{\circ} \mathrm{C}$ in upper extremities $\left(0.5 \pm 0.2^{\circ} \mathrm{C}\right.$ on the right upper and $0.7 \pm 0.2^{\circ} \mathrm{C}$ on the left upper side). The magnitude of the core temperature change after the impact was $0.2 \pm 0.1^{\circ} \mathrm{C}$. Statistically, there was no significant difference between the lower and upper extremities $(P=0.316)$; however, the degree of temperature change in lower extremities was significantly different from that of core temperature $(P=0.007)$.

\section{Comparison between Complete and Incomplete SCI Groups on the wake-up test}

There was no response during the wake-up test in the Complete SCI Group, and results were consistent with MEP results. The Incomplete SCI Group demonstrated a decrease in MEP signal by $>80 \%$ in all cases; however, animals in this group showed responses to the wakeup test, although their responses were variable. The comparison between the groups is shown in Table 3 .

\section{Histological examination}

In all cases of incomplete SCI, edema and subdural hematoma were observed macroscopically after SCI (Figure 7a). A varying range of spotting hemorrhage was observed in the central canal. However, definitive breakage of neural tissue at the ventral horn, where the autonomous nervous system is located, was not observed. The extent of damage of the white matter in the axial slice also varied, showing vacuoles and breaks in the connection between neural cells in the area of the lateral column (where the cortico-spinal tract is located) (Figure 7). Nevertheless, the amount and location of neural tissue damage were inconsistent. Since the duration of monitoring was $<48 \mathrm{~h}$, inflammatory cells were not observed around the hematoma. ${ }^{19}$

\section{DISCUSSION}

Our results indicate that there is a relationship between complete SCI and body surface temperature. This finding can be applied to aid the MEP monitoring as an ancillary measurement. Although, the major monitoring modality to indicate intraoperative SCI nowadays is TES-MEP, because of its proven accuracy and relatively continuous monitoring of motor nerves its use is limited by the complexity of pathophysiologic mechanisms of SCI, as indicated by false-positive and false-negative results. Furthermore, such limitations can cause uncertainties during spine surgeries, which interfere with important intraoperative decisions. Therefore, combining different monitoring techniques was necessary to appropriately define the intraoperative insult. As our research have suggested, body surface temperature change may aid in maximizing the benefits of MEP by overcoming the limitations, such as the inability to differentiate between simple concussion and severe irreversible neurological damage. This hypothesis was based on the fact that the surface temperature of the lower extremities elevates after SCI due to the damage in the descending pathway coming from the supraspinal center leading to sympathetic hypoactivity and vasodilatation. ${ }^{20}$ This possibility has been previously explored by Roehl K et al.,21 who measured the temperatures of the skin, and compared the injured dermatome with the dermatomes of intact spinal cord in SCI patients and reported that differences in surface temperature changes could be used to evaluate the level of SCI.

In our study, in the Incomplete SCI Group, the surface temperature of lower extremities elevated from 33.4 to $35.1^{\circ} \mathrm{C}$ (mean value of change: $1.7 \pm 0.5^{\circ} \mathrm{C}$ ). Considering the strict homeostatic temperature control in mammals, this is regarded as abnormal. Surface temperature in the lower extremities changed significantly compared with that of the upper extremities and core temperature $(P=0.004$ and 0.002 , respectively) (Figure 6a). This result is similar to a previous study that used the transected spinal cord on mouse model, which reported a 
$5.6^{\circ} \mathrm{C}$ and $4.0^{\circ} \mathrm{C}$ of temperature rise on the surface of the lower extremities and tail, respectively, for 5 days after SCI. ${ }^{11}$ Decreased cutaneous vasoconstrictor tone was suggested as the cause. In our study, the surface temperature of extremities elevated after complete SCI. However, in the Incomplete SCI Group, the surface temperature of the lower extremities changed from 34.9 to $35.4{ }^{\circ} \mathrm{C}$, which is not statistically different from the temperature change of the upper extremities $(P=0.316)$ (Figure 6b). Although, there was a statistical difference between the change in core temperature $\left(0.2^{\circ} \mathrm{C}\right)$ and that of the lower extremities $\left(0.5^{\circ} \mathrm{C}\right)$, this was thought to be insignificant, as it was within the normal range.

When the Complete and Incomplete SCI Groups were compared, the only differences found were the change in temperature in lower extremities and wake-up test score (Table 3). Considering minimal differences in biological and environmental conditions and in neurophysiologic states (that is, SCI on MEPs) between two groups, it can be assumed that the change in temperature in lower extremity mirrors the result of the wake-up tests after Complete and Incomplete SCI.

Histologicaly, the Incomplete SCI Group showed epidural hemorrhage in all cases (Figure 7) with varying amount and extent of hemorrhage in white and gray matter. Punctuate hemorrhage was found in all cases around the central canal and it spread out in the form of spokes of a wheel in the gray matter, although no definite neuronal injury was observed at ventral horn of gray matter. These findings were consistent with previous studies, which observed diffuse hemorrhage in gray matter in incompletely paralyzed subjects caused by weight drop impact. ${ }^{19}$ However, the authors did not see the disappearance of a large number of nerve cells in gray matter in incompletely paralyzed cases, that is, the Incomplete SCI Group (Figure 7), which was thought to be the factor that maintained the temperature of lower extremities. In white matter, a varying amount of linear breakage of neuronal connective tissue of the lateral funiculus was observed. It was assumed as the cause of loss of MEP signal and the unresponsiveness to the wake-up test or Von Frey filament stimulation in the Incomplete SCI Group.

The limitations of this study are as follows: (1) the extent of autonomic dysfunction differs depending on the injured level of spine that can have an influence on surface temperature. Thus, temperature changes following spinal injury at various levels need to be confirmed. Also it would not be possible to measure a temperature change of $1.7^{\circ} \mathrm{C}$ in a routine surgical practice by currently available methods. However, recently Roehl et al. ${ }^{21}$ established measurements of small increments in temperatures with the help of modern thermal cameras. The authors were also able to establish the level of paralysis and the neurological border between sensitive and non-sensitive body areas of 16 paralyzed patients. (2) To avoid analytical errors due to spinal shock that may occur during cord impact, different mechanism to produce SCI was used for each group. For the development of complete SCI, a cord transection method was used, instead of the weight drop method that was used for incomplete SCI. However, signs of spinal shock or effects on surface temperature following spinal shock as a result of using two separate methods to produce complete and incomplete SCI were not observed. (3) The controlled laboratory environment of the study is not akin to a clinical (intraoperative) situation, as many factors such as temperature and humidity cannot be kept constant in a clinical situation. (4) As scoring system for the locomotor evaluation of experimental animal was simplified and modified, and not subdivided into many levels, surface temperature change could not be evaluated according to the severity of the SCI. ${ }^{22}$ However, as the study aims to find the presence of a relationship between the SCI and change in surface temperature of lower extremity as a comprehensive preliminary study, more simple and concise scoring system was considered more appropriate, and therefore, used.

In summary, body surface temperature changes were found to be useful for assessing the completeness of cord injury in a rabbit SCI model. Exploration by further animal and clinical studies may help in ascertaining its role in optimization of the diagnostic efficacy of multimodal neuromonitoring for detection of impending neural injury.

\section{CONFLICT OF INTEREST}

The authors declare no conflict of interest.

\section{ACKNOWLEDGEMENTS}

This study was supported by a grant of the Korea Health Technology R\&D Project, Ministry of Health \& Welfare, Republic of Korea (HI11C0388). This study was performed under the approval of the IACUC (Institutional Animal Care and Use Committee, KU12129). All experimental procedures, protocols and treatment of experimental animals were performed under the control of the IACUC. This study was performed in the Korea University Guro Hospital.

\section{AUTHOR CONTRIBUTIONS}

SWS carried out the arrangement of the enrolled patients and drafted the manuscript as an assistant of JHY. YSP participated in the design of the study and performed the statistical analysis as an assistant of JHY. SWS carried out the arrangement of the enrolled patients and drafted the manuscript as an assistant of JHY. J-HL and BKP participated in the design of the study. CHH participated in the design and revision work of the study. JHY participated in the design of the study, statistical analysis and coordination, and drafted the manuscript as the main author. All authors read and approved the final manuscript.

\section{DISCLAIMER}

No benefits in any form have been or will be received from a commercial party related directly or indirectly to the subject of this manuscript.

1 Malhotra NR, Shaffrey Cl. Intraoperative electrophysiological monitoring in spine surgery. Spine 2010; 35: 2167-2179.

2 Balvin MJ, Song KM, Slimp JC. Effects of anesthetic regimens and other confounding factors affecting the interpretation of motor evoked potentials during pediatric spine surgery. Am J Electroneurodiagnostic Technol 2010; 50: 219-244.

3 Hong JY, Suh SW, Modi HN, Hur CY, Song HR, Park JH . False negative and positive motor evoked potentials in one patient: is single motor evoked potential monitoring reliable method? A case report and literature review. Spine 2010; 35: E912-E916.

4 Modi HN, Suh SW, Yang JH, Yoon JY. False-negative transcranial motor-evoked potentials during scoliosis surgery causing paralysis: a case report with literature review. Spine 2009; 34: E896-E900.

5 Bridwell KH, Dewald RL. The Textbook of Spinal Surgery, 3rd edn Wolters Kluwer/ Lippincott Williams \& Wilkins Health: Philadelphia, PA, USA 2011.

6 Langeloo DD, Journee HL, de Kleuver M, Grotenhuis JA. Criteria for transcranial electrical motor evoked potential monitoring during spinal deformity surgery A review and discussion of the literature. Neurophysiol Clin 2007; 37: 431-439.

7 Niederhofer $\mathrm{H}$. Effectiveness of the repetitive Transcranical Magnetic Stimulation (rTMS) of $1 \mathrm{~Hz}$ for Attention-Deficit Hyperactivity Disorder (ADHD). Psychiatr Danub 2008; 20: 91-92.

8 Meert TF, Vermeirsch HA. A preclinical comparison between different opioids: antinociceptive versus adverse effects. Pharmacol Biochem Behav 2005; 80: 309-326.

9 Kinoshita H, Yahagi S, Kasai T. Preparatory suppression of the human primary motor cortex induced by repetition of simple and choice reaction time tasks: a transcranial magnetic stimulation study. Brain Res 2007; 1184: 132-140.

10 MacDonald DB. Safety of intraoperative transcranial electrical stimulation motor evoked potential monitoring. J Clin Neurophysiol 2002; 19: 416-429.

11 Laird AS, Carrive P, Waite PM. Cardiovascular and temperature changes in spinal cord injured rats at rest and during autonomic dysreflexia. J Physiol 2006; 577: 539-548.

12 Metz GA, Curt A, van de Meent H, Klusman I, Schwab ME, Dietz V. Validation of the weight-drop contusion model in rats: a comparative study of human spinal cord injury. J Neurotrauma 2000; 17: 1-17. 
13 Yu K, Li J, Jia L, Bao J, Yuan W, Ye T et al. [The effects of graded spinal cord injuries on transcranial electric stimulation motor evoked potentials in the rat]. Zhonghua Wai Ke Za Zhi 1998; 36: 417-420.

14 Reece TB, Davis JD, Okonkwo DO, Maxey TS, Ellman PI, Li X et al. Adenosine A2A analogue reduces long-term neurologic injury after blunt spinal trauma. J Surg Res 2004; 121: 130-134.

15 Docherty B, Foudy C. Homeostasis part 3: temperature regulation. Nurs Times 2006; 102: $20-21$.

16 Tarlov IM, Klinger H. Spinal cord compression studies. II. Time limits for recovery after acute compression in dogs. AMA Arch Neurol Psychiatry 1954; 71: 271-290.

17 Basso DM, Beattie MS, Bresnahan JC. Graded histological and locomotor outcomes after spinal cord contusion using the NYU weight-drop device versus transection. Exp Neurol 1996; 139: 244-256.
18 Faul F, Erdfelder E, Buchner A, Lang AG. Statistical power analyses using G*Power 3.1: tests for correlation and regression analyses. Beh Res Methods 2009; 41 1149-1160.

19 White RJ. Pathology of spinal cord injury in experimental lesions. Clin Orthop Relat Res 1975; 112: 16-26.

20 Popa C, Popa F, Grigorean VT, Onose G, Sandu AM, Popescu M et al. Vascular dysfunctions following spinal cord injury. J Med Life 2010; 3: 275-285.

21 Roehl K, Becker S, Fuhrmeister C, Teuscher N, Futing M, Heilmann A. New, noninvasive thermographic examination of body surface temperature on tetraplegic and paraplegic patients, as a supplement to existing diagnostic measures. Spinal Cord 2009; 47: 492-495.

22 Onifer SM, Rabchevsky AG, Scheff SW. Rat models of traumatic spinal cord injury to assess motor recovery. ILAR J 2007; 48: 385-395. 